\title{
Incidence of first ever stroke during Hajj ceremony
}

\author{
Mahmoud Reza Azarpazhooh ${ }^{1 \dagger}$, Reza Bavarsad Shahripour ${ }^{2,3+}$, Moira K Kapral ${ }^{4}$, Naghmeh Mokhber ${ }^{5}$, Ali Shoeibi ${ }^{1}$, \\ Mohammad Taghi Farzadfard"', Mohammad Reza Rafati ${ }^{6}$, Amanda G Thrift', Negar Morovatdar ${ }^{8}$, \\ Seyed Aidin Sajedi ${ }^{3}$ and Amir Azarpazhooh ${ }^{4,9,10^{*}+}$
}

\begin{abstract}
Background: The Hajj Ceremony, the largest annual gathering in the world, is the most important life event for any Muslim. This study was designed to evaluate the incidence of stroke among Iranian pilgrims during the Hajj ceremony.

Methods: We ascertained all cases of stroke occurring in a population of 92,974 Iranian pilgrims between November 27, 2007 and January 12, 2008. Incidence and risk factors of the first ever stroke in Hajj pilgrims were compared, within the same time frame, to those of the Mashhad residents, the second largest city in Iran. Data for the latter group were extracted from the Mashhad Stroke Incidence Study (MSIS) database.

Results: During the study period, 17 first-ever strokes occurred in the Hajj pilgrims and 40 first-ever stroke strokes occurred in the MSIS group. Overall, the adjusted incidence rate of first ever stroke in the Hajj cohort was lower than that of the MSIS population (9 vs. 16 per 100,000). For age- and gender-specific subgroups, the Hajj stroke crude rates were in general similar to or lower than the general population of Mashhad, Iran, with the exception of women aged 35 to 44 years and aged $>75$ years who were at greater risk of having first-ever stroke than the non-pilgrims of the same age.

Conclusion: The first ever stroke rate among Iranian Hajj pilgrims was lower than that of the general population in Mashhad, Iran, except for females 35-44 or more than 75 years old. The number of events occurring during the Hajj suggests that Islamic countries should consider designing preventive and screening programs for pilgrims.
\end{abstract}

Keywords: Acute stroke, Hajj, Incidence

\section{Background}

The Hajj Ceremony, the largest annual gathering in the world, is the most important life event for any Muslim. For the majority of the pilgrims, this is a long-desired journey that may only happen once in their lifetime. Hence, it can be mentally stressful. Annually, millions of Muslims from various countries gather in the holy cities of Mecca and Medina in the Kingdom of Saudi Arabia to perform their religious rituals. Pilgrims are often in close contact,

\footnotetext{
* Correspondence: amir.azarpazhooh@utoronto.ca

tEqual contributors

${ }^{4}$ Institute of Health Policy, Management and Evaluation, Faculty of Medicine, University of Toronto, Ontario, Canada

${ }^{9}$ Discipline of Dental Public Health, Faculty of Dentistry, University of Toronto, Room 515-C, 124 Edward St, Toronto, ON M5G 1G6, Canada

Full list of author information is available at the end of the article
}

and are at increased risk of viral infections, particularly upper respiratory tract infections [1-5].

Despite the importance of Hajj, there are scant data on the incidence of important diseases such as vascular conditions that occur during the pilgrimage. Quantifying rates of cardiovascular disease during Hajj is important for Islamic countries [6-9]. If the stroke rate is high during Hajj, the Kingdom of Saudi Arabia may need to reevaluate its health system infrastructure and hospitals for stroke treatment. Moreover, Islamic countries may need to develop vascular preventive and screening programs for pilgrims. In addition, because of high reported rates of infection and psychological stress, the Hajj pilgrimage brings a unique opportunity to evaluate such risk factors on the occurrence of stroke [6].

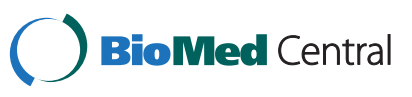




\section{Objective}

The objectives of this study were: 1) to determine the incidence of first-ever stroke among Iranian pilgrims during the Hajj ceremony, and 2) to compare the stroke incidence rate to a proxy estimate of Iran's national stroke rate.

\section{Methods}

\section{Study design and source of data}

The study was approved by the Ethics Committee of the Hajj and Pilgrimage Organization of Iran and Mashhad University of Medical Sciences (protocol No. 2195390). The study participants were 92,974 Iranian pilgrims who attended the Hajj ceremony between November 27, 2007 and January 12, 2008. These pilgrims were from different socioeconomic backgrounds and different rural and urban areas. According to the guidelines of the Hajj and Pilgrimage Organization of Iran, candidates with severe dementia, uncontrollable cancer, severe disabling stroke and recent (less than 3 months) myocardial infarction are not eligible to participate in the pilgrimage. After the initial eligibility screening, pilgrims were gathered as 605 groups (caravans, each including 100-200 pilgrims) and were evaluated before, during, and after the Hajj ceremony. Before and during the trip, a General Practitioner (GP) was assigned to each caravan.

Before the trip, the GPs received extensive training in the medical protocol of the Hajj and Pilgrimage Organization of Iran. They were responsible for registering past medical conditions, including the history of previous stroke, and evaluating the health status eligibility of the pilgrims in their assigned caravans. This information was recorded in the patient health chart kept by the GP of each caravan. During the Hajj pilgrimage, the GPs were the first point of contact for the pilgrims in case of medical concerns. The GPs then referred cases with neurological findings to the two permanent hospitals of the Iran Red Crescent Society in the Holy cities of Mecca and Medina, under the care of the study neurologist (MRA). When imaging was required, the cases were referred to the local Saudi's Hospitals. All patients were provided with free access to hospital care and laboratory tests. After the Hajj ceremony, all Iranian pilgrims were fully covered by a private healthcare company for any follow up visits, admission and treatment of diseases related to the Hajj period. The health data of the pilgrims, including their demographic characteristics, past medical history, medical events during and after the Hajj ceremony, decisions taken by GPs and subsequent referrals, cause of admission and outcome were registered in the central data bank of the Hajj and Pilgrimage Organization of Iran. We received the de-identified dataset and ascertained all cases of first ever-stroke in Hajj pilgrims through the retrospective evaluation of pilgrims' medical records. We also ascertained stroke events in non-pilgrims from the Mashhad Stroke Incidence Study (MSIS), our recently published population-based prospective cohort study [10]. The MSIS registered all strokes occurring in a population of 450,229 residents of the City of Mashhad (the second largest city in Iran) were registered during a 12-month period (2006-2007) [10].

\section{Variables and outcome measures}

The primary outcome in this study was the incidence of stroke, defined according to the World Health Organization as rapidly developing signs of focal or global disturbance of cerebral function, lasting for more than 24 hours (unless interrupted by surgery or death) with no apparent cause other than a vascular origin $[11,12]$. The First-Ever Stroke was defined as a stroke occurring for the first time during a patient's lifetime [12]. Previous stroke was determined using all available information including hospital records, neuroimaging results and/or self- or family-reported data. Neuroimaging was used to classify cases with definite strokes into ischemic stroke, intra-cerebral haemorrhage, or subarachnoid haemorrhage. As per the most recent consensus definition the American Heart Association/American Stroke Association (AHA/ASA), we included cerebral venous thrombosis (CVT) as stroke only if patients had infarction or hemorrhage in localized area of brain because of thrombosis of a cerebral venous structure [12].

An "undetermined stroke" was defined as a stroke in which a patient had not undergone CT scanning within 28 days of the onset of symptoms and an autopsy had not been performed [12].

A "possible stroke" was considered as any acute episode of neurological disturbance that was suggestive of stroke but there was insufficient information to establish whether or not the symptoms and duration $(<24$ hours or $>24$ hours) fully met the World Health Organization definition of the definite stroke [13]. A "CT only" stroke was defined when the patient had no clinical signs and symptoms of stroke, but neuroimaging identified changes compatible with stroke [13]. "Possible" and "CT only" strokes were excluded from the study. We also excluded CVT cases for whom the symptoms or signs were caused by reversible edema without infarction or hemorrhage [12].

The following risk factors were assessed for those patients diagnosed with first-ever stroke: gender, age, stressful periods (i.e., mentally or emotionally disruptive or upsetting situations such as getting lost, losing money, missing ritual rules, etc.), current smoking status, and past history of hypertension, diabetes, hyperlipidaemia, transient ischemic attack, ischemic heart disease, and atrial fibrillation. For our second objective, we regarded the Hajj pilgrims as the exposed group and compared them to the non-exposed population, derived 
from the data source of the MSIS stroke incidence study, in the manner of a historical cohort. From the MSIS database, the first-ever stroke cases during the exact period of the Hajj ceremony were extracted.

\section{Statistical analysis}

The first-ever stroke crude incidence rates were calculated using the total of Iranian Hajj pilgrims and the MSIS subjects (above age of 15, in the same time frame), respectively as the denominator for the Hajj group and the MSIS group. To calculate standardized incidence rates, we used direct method of adjustment with SEGI world population and IRAN 2006 standard population. We calculated the adjusted incidence rates for each population and by gender. Due to the different population pyramids of Hajj pilgrims and Mashhad residents, the crude incidence rates were compared only for stratified age and gender groups between two cohorts. The 95\% confidence intervals (CI) were calculated for adjusted rates on the assumption of a normal distribution for counts greater than 100 and a Poisson distribution for counts less than 100. The incidence rates were reported as number of cases per 100,000 population per month. Proportions were compared by the Chi-square test or Fisher's exact test when appropriate. Students T-test was used to compare continuous variables. All statistical tests were undertaken using SPSS v13.0 software. The significance was set at $\mathrm{P}<0.05$ (two-sided).

\section{Results}

\section{Descriptive results}

The age distribution of the Hajj pilgrims and the MSIS population is presented in Figure 1. While $55 \%$ of the MSIS population was less than 35 years of age, the majority of the pilgrims (75\%) were between the ages of 35 and 64 years. During the study period, GPs referred 19 cases suspected of having stroke. A final diagnosis of first-ever stroke by the study neurologist was made for 17 of these cases (5 men and 12 women). No case was suspected of "possible" or "CT only" strokes. Thirteen patients had ischemic stroke (including two cases with cerebral vein thrombosis); two had intra-cerebral haemorrhage; and the other two had undetermined stroke. CT or MRI of the brain was performed in 15 cases. Within the same time period, 40 cases $(23$ men and 17 women) with a final diagnosis of first-ever stroke were registered in the MSIS database. These included 29 cases of ischemic stroke, 10 cases of haemorrhagic stroke and one case of undetermined stroke. The baseline characteristics and risk factors of first-ever stroke patients were similar between the two groups (Table 1).

\section{First-ever stroke incidence}

Table 1 summarizes the age- and gender- specific crude incidence rates as well as the SEGI- and IRAN 2006adjusted rates of first-ever stroke in the two cohorts. For the age- and gender- specific crude incidence rates, we noted a lower first-ever stroke rates in the Hajj group for all strata, except for women aged 35 to 44,75 to 84 and greater than 85 years. In these subgroups, stroke rates were significantly higher in the Hajj than in the MSIS cohort. Overall, the SEGI-adjusted incidence rate in the Hajj cohort was lower than that of the MSIS population (7; $95 \% \mathrm{CI}$ : $4-11$ vs. 13 ; $95 \% \mathrm{CI}$ : $9-17$ per $100,000$ per month; $\mathrm{P}=0.03)$. This pattern was similar for males (4; 95\% CI:0.5-7 vs.14; 95\% CI: $8-20$, per 100,000 per month ; $\mathrm{P}=0.02$ ). However, the adjusted incidence rate of first-ever stroke in the Hajj female population was not statistically different from the MSIS population (13; $95 \%$ CI: $6-21$ vs. 11 ; $95 \%$ CI:6-17 per 100,000 per month; $\mathrm{P}=0.07$ ). The IRAN 2006-adjusted rates followed a pattern similar to those of the SEGIadjusted rates.

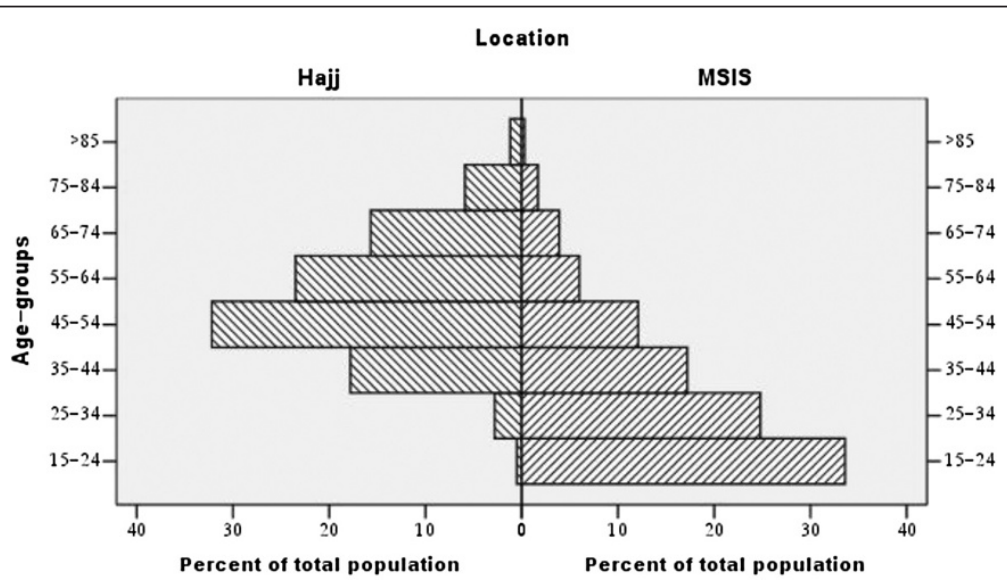

Figure 1 Distribution of Hajj and MSIS cohorts by age groups. 
Table 1 Age- and gender -specific Incidence Rates (per 100,000 Population per month) and adjusted incidence rates for First-Ever Stroke in Hajj (2007) compared with the data from MSIS within the same period

\begin{tabular}{|c|c|c|c|c|c|c|c|}
\hline \multirow[t]{2}{*}{ Age (years) } & \multicolumn{3}{|c|}{ Hajj subjects } & \multicolumn{3}{|c|}{ MSIS subjects } & \multirow[t]{2}{*}{$P$ value } \\
\hline & No. & Population at risk & Incidence rate $(95 \% \mathrm{Cl})$ & No. & Population at risk & Incidence rate $(95 \% \mathrm{Cl})$ & \\
\hline \multicolumn{8}{|l|}{ Both genders } \\
\hline $15-24$ & 0 & 514 & - & 0 & 112588 & - & - \\
\hline $25-34$ & 0 & 4110 & - & 2 & 83131 & $2(0-5)$ & - \\
\hline $35-44$ & 2 & 19397 & $10(4-16)$ & 1 & 57743 & $2(0-5)$ & $0.021^{\ddagger}$ \\
\hline $45-54$ & 3 & 29973 & $10(4-16)$ & 5 & 40596 & $12(5-20)$ & $0.67^{\dagger}$ \\
\hline $55-64$ & 3 & 21584 & $14(7-21)$ & 5 & 20096 & $25(15-36)$ & $0.07^{\dagger}$ \\
\hline $65-74$ & 5 & 12568 & $40(28-52)$ & 11 & 13103 & $84(66-102)$ & $0.001^{\dagger}$ \\
\hline $75-84$ & 3 & 4033 & $74(57-91)$ & 13 & 5944 & $219(190-248)$ & $0.001^{\dagger}$ \\
\hline$>85$ & 1 & 739 & $135(112-158)$ & 3 & 1147 & $262(230-294)$ & $0.001^{\dagger}$ \\
\hline Total & 17 & 92974 & $18(10-26)$ & 40 & 334348 & $12(5-19)$ & \\
\hline Standardized to SEGI* & & & $7(4-11)$ & & & $13(9-17)$ & 0.03 \\
\hline Standardized to IRAN $\wedge$ & & & $7(5-9)$ & & & $12(8-15)$ & 0.04 \\
\hline \multicolumn{8}{|l|}{ Males } \\
\hline $15-24$ & 0 & 234 & - & 0 & 55458 & - & - \\
\hline $25-34$ & 0 & 1303 & - & 1 & 42266 & $2(0-5)$ & - \\
\hline $35-44$ & 0 & 8102 & - & 0 & 29202 & - & - \\
\hline $45-54$ & 2 & 14646 & $14(7-21)$ & 3 & 20681 & $15(7-23)$ & $0.85^{\dagger}$ \\
\hline $55-64$ & 2 & 10704 & $19(10-28)$ & 2 & 10146 & $20(11-29)$ & $0.87^{+}$ \\
\hline $65-74$ & 0 & 7140 & - & 5 & 6820 & $73(56-90)$ & - \\
\hline $75-84$ & 1 & 2710 & $37(25-49)$ & 10 & 3109 & $322(287-357)$ & $0.001^{\dagger}$ \\
\hline$>85$ & 0 & 547 & - & 2 & 556 & $360(323-397)$ & - \\
\hline Totals & 5 & 45419 & $11(4-18)$ & 23 & 168238 & $14(7-21)$ & \\
\hline Standardized to SEGI* & & & $4(0.5-7)$ & & & $14(8-20)$ & 0.02 \\
\hline Standardized to IRAN $\wedge$ & & & $4(0.5-8)$ & & & $17(10-24)$ & 0.01 \\
\hline \multicolumn{8}{|l|}{ Females } \\
\hline $15-24$ & 0 & 280 & - & 0 & 57130 & - & - \\
\hline $25-34$ & 0 & 2807 & - & 1 & 40865 & $2(0-5)$ & - \\
\hline $35-44$ & 2 & 11295 & $18(10-26)$ & 1 & 28541 & $4(0-8)$ & $0.003^{\dagger}$ \\
\hline $45-54$ & 1 & 15327 & $7(2-12)$ & 2 & 19915 & $10(4-16)$ & $0.46^{\dagger}$ \\
\hline $55-64$ & 1 & 10880 & $9(3-15)$ & 3 & 9950 & $30(19-41)$ & $0.001^{\dagger}$ \\
\hline $65-74$ & 5 & 5428 & $92(73-111)$ & 6 & 6283 & $95(76-114)$ & $0.82^{\dagger}$ \\
\hline $75-84$ & 2 & 1323 & $151(127-175)$ & 3 & 2835 & $106(86-126)$ & $0.005^{\dagger}$ \\
\hline$>85$ & 1 & 192 & $521(476-566)$ & 1 & 591 & 169 (144-194) & $0.001^{\dagger}$ \\
\hline Total females & 12 & 47555 & $25(15-35)$ & 17 & 166110 & $10(4-16)$ & \\
\hline Standardized to SEGI* & & & $13(6-21)$ & & & $11(6-17)$ & 0.07 \\
\hline Standardized to IRAN^ & & & $15(6-23)$ & & & $12(7-18)$ & 0.08 \\
\hline
\end{tabular}

MSIS: Mashhad Stroke Incidence Study [11].

${ }^{\dagger}$ Chi-square test; \#Fisher exact test; N/A: Not applicable.

*adjusted by age and sex to the world (SEGI) population.

$\wedge$ adjusted by age and sex to the Iran population.

\section{Discussion}

We investigated the incidence of stroke among Iranian pilgrims during the 2007 Hajj ceremony, and found that the overall incidence was lower as compared to what was seen in the general population of Mashhad, Iran. This pattern is likely attributable to the pre-pilgrimage medical screening performed by the Hajj and Pilgrimage Organization of Iran. The screening program for the 
Hajj is simple, comprising evaluation of blood pressure, diabetes and other vascular risk factors. In suspected cases of vascular disorders, electrocardiography is performed with referral for cardiology or neurology consultation. Candidates with severe dementia, uncontrollable cancer, severe disabling stroke and recent (less than 3 months) myocardial infarction are excluded from participation in the Hajj ceremony. Therefore, it can be argued that such enhanced screening system may have reduced to some degree the stroke risk factors by selecting individuals devoid of severe and life-threatening disease. The finding implies a research hypothesis whether population-based screening programs would reduce stroke in communities. This simple screening could be tested in the general population with the aim of treating these risk factors to prevent vascular events. It is also possible that the pilgrims with the knowledge of having the upcoming trip would be more conscious about their health status. In other words, people participating in the Hajj are healthier than the general population, similar to what is observed in the healthy migrant effect [14].

Previous small-scale studies reported that $20-35 \%$ of hospital admissions during Hajj were related to old age and occurred in patients with associated cardiovascular conditions [15-18]. To the best of our knowledge no study has thus far assessed the incidence of stroke among the Hajj participants. In this present study, we found that stroke risk in Hajj pilgrims was increased relative to the general population of Mashhad, Iran in some subgroups of women, including those aged 33 to 44 years and those aged over 75 years. We have previously reported a greater risk of CVT in Hajj pilgrim females [18-38]. Young Muslim women often take oral contraceptives in the short term during religious ceremonies to avoid menstruation. This is because menstruation would cancel the sacred state of Ihram, into which a Muslim must enter in order to perform the religious rituals. In this present study, we had two cases with CVT who fell in the stroke criteria of the AHA/ASA [12]. It is possible that the use of oral contraceptives during the Hajj contributed to the increased risk of stroke in women aged 35 to 44 years compared to the general population of Mashhad, Iran. It needs to be noted that some epidemiological studies of stroke did not include CVT. Ischemic stroke and CVT are important types of stroke and comply with the clinical definition of stroke. In 2013 Updated Definition of Stroke for the 21st Century, the American Heart Association/American Stroke Association included CVT with specific criteria (infarction or hemorrhage in brain because of thrombosis of a cerebral venous structure) as stroke [9]. Therefore, we included these two cases that fell under these specific criteria.

In addition, pilgrims are subjected to high rates of physical and mental stress as well as infection, in particular upper respiratory tract infections) [19]. Previous studies have suggested that recent bacterial or viral infections, particularly in the preceding week, are a risk factor for stroke [19-21]. Some studies have also shown an association between respiratory tract infection epidemics and death due to cardiovascular disorders [19], and reductions in cardiovascular mortality and stroke after influenza vaccination [22-24] and control of epidemic respiratory diseases [25-27]. While such infections, inflammatory processes and psychological stress may be predisposing risk factors for stroke and cardiovascular disease, their exact role remains unclear [28-33].

Within the Hajj pilgrims, an increased risk of first-ever stroke was noted in females aged over 65 years compared to their male counterparts. The reasons for this are uncertain, and may include baseline differences in stroke risk factors or comorbid conditions. Other potential explanations include gender differences in the neurobiological responses to psychological stress. It has been shown that brain locations activated in response to psychological stress, the subsequent systemic responses and the duration of the effect of the stress are different in men and women $[22,34,35]$. That said, the existing literature regarding psychological distress and risk of stroke is scant, and presents varied findings [22-36].

This study has some limitations. Any occurrence of stroke would have health planning implications; however, in order to have a comparable group with the MSIS subjects, we evaluated only first ever strokes rather than all strokes. Although the pre-pilgrimage medical screening may have resulted in overall lower first-ever stroke rates in Iranian pilgrims, it may have imposed an unavoidable selection bias, once comparing the Hajj pilgrims with the general population of Mashhad, Iran. The latter group comprises those with diverse socioeconomical status and underlying factors that may affect their health. Mashhad is the second populous metropolitan of Iran and a religious centre that attracts many migrants from all parts of the country. The MSIS has been so far the only comprehensive population-based study of stroke in Iran. Although the result of this study cannot readily be generalized to the whole country, other unpublished local data suggest comparable cerebrovascular risk profile in other cities, similar to what was found in the MSIS. This gives us some confidence that Mashhad can be regarded as representative of the urban population of Iran and the MSIS results can provide a good proxy of the national incidence of urban population, but not the rural population. It should also be noted that the baseline information and risk profile were only available from the Hajj pilgrims with first-ever stroke, not all the pilgrims. Therefore, the comparison between demographic and premorbid risk factors of all Haji pilgrims and the general population of Mashhad, Iran was not possible. In addition, 
we were unable to evaluate the processes and outcomes of care for Hajj pilgrims with stroke.

Despite these limitations, this study was strengthened by the fact that we accessed the medical information of more than 90,000 Iranian pilgrims who were closely monitored during the Hajj ceremony. To the best of our knowledge, this is the first stroke epidemiological study among Hajj pilgrims, gathering at the largest annual event in the world. Islam has 1.6 billion followers in the world, comprising over $23 \%$ of the world population [39]. Due to the religious rules, attending in Hajj ceremony, at least for once, is an aim for Muslims. Iran is one of the 22 Member States of the WHO Eastern Mediterranean Region (EMRO). These Member States, with a population of nearly 583 million people, are predominantly Muslims [40]. Therefore, the findings of this study may be considered of value for the EMRO countries in preparation for the Hajj ceremony, a very large population.

\section{Conclusions}

In conclusion we found that the first ever stroke rate among Iranian Hajj pilgrims was lower than that of the general population in Mashhad, Iran, except for females 35-44 or more than 75 years old. Although the results cannot be generalized to pilgrims from other countries, they highlight the importance of preventive and screening programs aimed to reduce vascular risk factors, stress, and respiratory infections, and the need for the Kingdom of Saudi Arabia to evaluate the infrastructure of its healthcare system and hospitals to ensure that appropriate medical care including acute stroke treatment is available during the Hajj.

\section{Competing interests}

The authors declare that they have no competing interests.

\begin{abstract}
Authors' contributions
MRA has made substantial contributions to the study conception, design, and implementation. He revised the manuscript critically for important intellectual content. RBS participated in the study design and has been involved in drafting and revising the manuscript critically for important intellectual content. MKK and AGT helped in revising the manuscript critically for important intellectual content according to its result. NM and MRR helped to design and coordinate the study. AS and MTF participated in the study design and data collection. SAS and NMD performed the statistical analysis and has been involved in drafting the manuscript. AA has made substantial contributions to conception and design, helped to plan for statistical analysis, and has been involved in drafting the manuscript and revising it critically for important intellectual content. All authors read and approved the final manuscript.
\end{abstract}

\section{Acknowledgement}

This study was supported by Iranian Red Crescent Society and Hajj and Pilgrimage Organization of Iran.

\section{Author details}

'Department of Neurology, Ghaem Hospital, Mashhad University of Medical Sciences, Mashhad, Iran. ${ }^{2}$ Comprehensive Stroke Center, University of Alabama Hospital, Birmingham, AL, USA. ${ }^{3}$ Department of Neurology, Golestan Hospital, Ahvaz University of Medical Sciences, Ahwaz, Iran.
${ }^{4}$ Institute of Health Policy, Management and Evaluation, Faculty of Medicine, University of Toronto, Ontario, Canada. ${ }^{5}$ Department of Neuropsychology, Ibn-e-Sina Hospital, Mashhad University of Medical Science, Mashhad, Iran. ${ }^{6}$ Department of Internal Medicine, Red Crescent Society, Tehran, Iran. ${ }^{7}$ Department of Medicine, Southern Clinical School, Monash University, Melbourne, and National Stroke Research Institute, Florey Neuroscience Institutes, Heidelberg Heights, Victoria, Australia. ${ }^{8}$ Health System Research Committee, Treatment Affairs of vice chancellery, Mashhad University of Medical Sciences, Mashhad, Iran. ${ }^{9}$ Discipline of Dental Public Health, Faculty of Dentistry, University of Toronto, Room 515-C, 124 Edward St, Toronto, ON M5G 1G6, Canada. ${ }^{10}$ Toronto Health Economics and Technology Assessment Collaborative, University of Toronto, Toronto, Canada.

Received: 18 November 2012 Accepted: 27 November 2013

Published: 5 December 2013

\section{References}

1. Greenwood DC, Muir KR, Packham CJ, Madeley RJ: Coronary heart disease: a review of the role of psychosocial stress and social support. J Public Health Med 1996, 18(2):221-231. Epub 1996/06/01.

2. Nieto FJ: Infections and atherosclerosis: new clues from an old hypothesis? Am J Epidemiol 1998, 148(10):937-948. Epub 1998/11/26.

3. Grau AJ, Buggle F, Heindl S, Steichenwiehn C, Banerjee T, Maiwald M, et al: Recent infection as a risk factor for cerebrovascular ischemia. Stroke 1995, 26(3):373-379.

4. Grau AJ, Buggle F, Steichenwiehn C, Heindl S, Banerjee T, Seitz R, et al: Clinical and biochemical-analysis in infection-associated stroke. Stroke 1995, 26(9):1520-1526.

5. Mattila KJ, Valtonen W, Nieminen MS, Asikainen S: Role of infection as a risk factor for atherosclerosis, myocardial infarction, and stroke. Clin Infect Dis 1998, 26(3):719-734. Epub 1998/04/03.

6. Meysamie A, Ardakani HZ, Razavi SM, Doroodi T: Comparison of mortality and morbidity rates among Iranian pilgrims in Hajj 2004 and 2005. Saudi Med J 2006, 27(7):1049-1053.

7. Jakovljevic D, Salomaa V, Sivenius J, Tamminen M, Sarti C, Salmi K, et al: Seasonal variation in the occurrence of stroke in a finnish adult population. The FINMONICA stroke register. Finnish monitoring trends and determinants in cardiovascular disease. Stroke 1996, 27(10):1774-1779. Epub 1996/10/01.

8. Everson SA, Roberts RE, Goldberg DE, Kaplan GA: Depressive symptoms and increased risk of stroke mortality over a 29-year period. Arch Intern Med 1998, 158(10):1133-1138. Epub 1998/05/30.

9. Simonsick EM, Wallace RB, Blazer DG, Berkman LF: Depressive symptomatology and hypertension-associated morbidity and mortality in older adults. Psychosom Med 1995, 57(5):427-435. Epub 1995/09/01.

10. Azarpazhooh MR, Etemadi MM, Donnan GA, Mokhber N, Majdi MR, Ghayour-Mobarhan M, et al: Excessive incidence of stroke in iran: evidence from the Mashhad stroke incidence study (MSIS), a populationbased study of stroke in the Middle East. Stroke 2010, 41(1):e3-e10. Epub 2009/11/21.

11. Colantonio A, Kasl SV, Ostfeld AM: Depressive symptoms and other psychosocial factors as predictors of stroke in the elderly. Am J Epidemiol 1992, 136(7):884-894.

12. Sacco RL, Kasner SE, Broderick JP, Caplan LR, Connors JJ, Culebras A, et al: An updated definition of stroke for the 21st century: a statement for healthcare professionals from the American heart association/American stroke association. Stroke 2013, 44(7):2064-2089. Epub 2013/05/09.

13. Marnane M, Duggan CA, Sheehan OC, Merwick A, Hannon N, Curtin D, et al: Stroke subtype classification to mechanism-specific and undetermined categories by TOAST, A-S-C-O, and causative classification system: direct comparison in the North Dublin population stroke study. Stroke 2010, 41(8):1579-1586. Epub 2010/07/03.

14. Fennelly K: The "healthy migrant" effect. Minn Med 2007, 90(3):51-53. Epub 2007/04/17.

15. Al-Ghamdi SM, Akbar HO, Qari YA, Fathaldin OA, Al-Rashed RS: Pattern of admission to hospitals during muslim pilgrimage (Hajj). Saudi Med J 2003, 24(10):1073-1076. Epub 2003/10/28.

16. W.H A: The prevalence of cardiovascular disease and role of protective measures among Hajj pilgrims 1432. Pak J Pharmacol 2012, 29:29-34.

17. Khan NAIA, Ahmad MS, El-Sayed FM, Bachal ZA, Abbas TG: Pattern of medical diseases and determinants of prognosis of hospitalization 
during 2005 Muslim pilgrimage Hajj in a tertiary care hospital. A prospective cohort study. Saudi Med J 2006, 27:1373-1380.

18. Azarpazhooh MR, Rafi S, Etemadi MM, Khadem N, Fazlinejad A: The relation between short-term oral contraceptive consumption and cerebrovascular, cardiovascular disorders in Iranian women attending Hajj. Saudi Med J 2008, 29(7):1024-1027.

19. Alzeer AH: Respiratory infection in haj ceremony. Ann Thorac Med 2009, 4:50-53.

20. Yamamoto: Perceived mental stress and mortality from cardiovascular disease among japanese men and women: the Japan collaborative cohort study for evaluation of cancer risk sponsored by monbusho. Circulation 2002, 106:1229-1236.

21. Andre-Petersson L, Engstrom G, Hagberg B, Janzon L, Steen G: Adaptive behavior in stressful situations and stroke incidence in hypertensive men - Results from prospective cohort study "men born in 1914" in Malmo, Sweden. Stroke 2001, 32(8):1712-1717.

22. Harmsen $\mathrm{P}$, Rosengren $\mathrm{A}$, Tsipogianni A, Wilhelmsen L: Risk factors for stroke in middle-aged men in Goteborg, Sweden. Stroke 1990, 21(2):223-229. Epub 1990/02/01.

23. Macko RF, Ameriso SF, Barndt R, Clough W, Weiner JM, Fisher M: Precipitants of brain infarction. Roles of preceding infection/ inflammation and recent psychological stress. Stroke 1996, 27(11):1999-2004. Epub 1996/11/01.

24. WassertheilSmoller S, Applegate WB, Berge K, Chang CJ, Davis BR, Grimm R, et al: Change in depression as a precursor of cardiovascular events. Arch Intern Med 1996, 156(5):553-561.

25. Zeller JA LA, Eschenfelder CC, Zunker P, Deuschl G: Platelet-leukocyte interaction and platelet activation in acute stroke with and without preceding infection. Arterioscler Thromb Vasc Biol 2005, 25:1519-1523.

26. Laurila A, Bloigu A, Nayha S, Hassi J, Leinonen M, Saikku P: Chronic Chlamydia pneumoniae infection is associated with a serum lipid profile known to be a risk factor for atherosclerosis. Arteriosd Throm Vas 1997 17(11):2910-2913.

27. Mayr M, Kiechl S, Willeit J, Wick G, Xu Q: Infections, immunity, and atherosclerosis: associations of antibodies to Chlamydia pneumoniae, Helicobacter pylori, and cytomegalovirus with immune reactions to heat-shock protein 60 and carotid or femoral atherosclerosis. Circulation 2000, 102(8):833-839. Epub 2000/08/23.

28. Housworth J, Langmuir AD: Excess mortality from epidemic influenza, 1957-1966. Am J Epidemiol 1974, 100(1):40-48. Epub 1974/07/01.

29. Alling DW, Blackwelder WC, Stuart-Harris CH: A study of excess mortality during influenza epidemics in the United States, 1968-1976. Am J Epidemiol 1981, 113(1):30-43. Epub 1981/01/01.

30. May MMP, Stansfeld S, Ben-Shlomo Y, Gallacher J, Yarnell J, et al: Does psychological distress predict the risk of ischemic stroke and transient ischemic attack? The Caerphilly study. Stroke 2002, 33:7-12.

31. Bova IY, Bornstein NM, Korczyn AD: Acute infection as a risk factor for ischemic stroke. Stroke 1996, 27(12):2204-2206.

32. Grau AJ, Buggle F, Becher H, Zimmermann E, Spiel M, Fent T, et al: Recent bacterial and viral infection is a risk factor for cerebrovascular ischemia - Clinical and biochemical studies. Neurology 1998, 50(1):196-203.

33. House A, Dennis M, Mogridge L, Hawton K, Warlow C: Life events and difficulties preceding stroke. J Neurol Neurosurg Psychiatr Supp/1990, 53(12):1024-1028. Epub 1990/12/01.

34. Wang J, Korczykowski M, Rao H, Fan Y, Pluta J, Gur RC, et al: Gender difference in neural response to psychological stress. Soc Cogn Affect Neurosci 2007, 2(3):227-239. Epub 2007/09/18.

35. Andre-Petersson L, Engstrom G, Hagberg B, Janzon L, Rosvall M: Social support at work and the risk of myocardial infarction and stroke in women and men. Soc Sci Med 2007, 64(4):830-41. Epub 2006 Nov 22.

36. Bainton D, Jones GR, Hole D: Influenza and ischaemic heart disease-a possible trigger for acute myocardial infarction? Int J Epidemiol 1978, 7(3):231-239. Epub 1978/09/01.

37. Saidee MFM, Sasannejad P, Mellat Ardakani A, Azarpazhooh M: The relation between short course oral contraceptive consumption and cerebral vein thrombosis in Ramadan. Iran J Neurol 2008, 7(23):260-523.

38. Sasannejad PAA, Velayati A, Shoeibi A, Saeidi M, Foroughipour M, et al: Cerebral vein thrombosis in women using short course oral contraceptive consumption. Iran J Reprod Med 2012, 10(6):537-542.
39. The Global Religious Landscape: A Report on the Size and Distribution of the World's Major Religious Groups as of 2010. Washington, D.C: Pew Research Center's Forum on Religion \& Public Life; 2012. Available from: www. pewforum.org/files/2012/12/globalReligion-full.pdf.

40. World Health Organization Regional Office for the Eastern Mediterranean (EMRO): http://www.emro.who.int/entity/about-us/index.html.

doi:10.1186/1471-2377-13-193

Cite this article as: Azarpazhooh et al.: Incidence of first ever stroke during Hajj ceremony. BMC Neurology 2013 13:193.

\section{Submit your next manuscript to BioMed Central and take full advantage of:}

- Convenient online submission

- Thorough peer review

- No space constraints or color figure charges

- Immediate publication on acceptance

- Inclusion in PubMed, CAS, Scopus and Google Scholar

- Research which is freely available for redistribution

Submit your manuscript at www.biomedcentral.com/submit
C Biomed Central 